http://www.jfas.info

\title{
HEART ABNORMALITY DETECTION BY USING ARTIFICIAL NEURAL NETWORK
}

F. R. Hashim ${ }^{1, *}$, J. Adnan $^{1}$, M. M. Ibrahim², M. T. Ishak ${ }^{1}$, M. F. M. $\operatorname{Din}^{1}$, N. G. N. Daud ${ }^{1}$ and Z. I. Rizman ${ }^{3}$

${ }^{1}$ Faculty of Electrical and Electronic Engineering, National Defense University of Malaysia, Kuala Lumpur, Malaysia

${ }^{2}$ Faculty of Electronic and Computer Engineering, Universiti Teknikal Melaka, Melaka, Malaysia

${ }^{3}$ Faculty of Electrical Engineering, Universiti Teknologi MARA, 23000 Dungun, Terengganu, Malaysia

Published online: 10 September 2017

\begin{abstract}
The detection of heart abnormality activity of a patient is important when an abnormal symptom occurred. This project is aimed to detect the disease by using Hybrid Multilayer Perceptron (HMLP) network. Several data from the ECG signal is extracted to be set as input parameters. In order to get the best result, the proposed training algorithm of HMLP network is by using Modified Recursive Prediction Error (MRPE). The result obtained is then compared with other training algorithm method to determine the best performance. The HMLP network is expected to give better performance compared with other network classifiers.
\end{abstract}

Keywords: heart abnormality; radial basis function; electrocardiogram.

Author Correspondence, e-mail: fakroul@upnm.edu.my

doi: http://dx.doi.org/10.4314/jfas.v9i3s.1 


\section{INTRODUCTION}

Heart abnormality activity or heart disease is a very common disease that people in the world have, especially in Malaysia. Heart abnormality or heart disease will be occurred when the blood flow into the heart is blocked by a blood clot [1]. The present of the blood clot will make the artery which connected to the heart become narrow. If the blood clots totally block the main artery to the heart, blood supply will eventually stop flowing into it. This will make the heart 'starved' for oxygen, because oxygen is important for pumping the blood back to the whole body. In a short time, the heart muscle will become weak, and supply less blood to the whole body because of lack of oxygen. This causes the heart attack.

One of the most effective ways to detect the heart abnormality is by using electrocardiogram (ECG). ECG is the important source for doctor to detect the any cardiac abnormality or heart disease. It works as a detector of any electrical activity on the surface of a body that generated by the heart [2]. The measurement that is being recorded by ECG is collected by placing several skin electrodes at some specific area of a body.

However, there are known pitfalls in electrocardiography [2]. In particular, an ECG may appear normal despite the presence of serious heart problems. In fact, because the ECG is just a record at one point in time like snapping a photograph, a patient with a normal ECG may nonetheless (rarely) develop a major change indicative of a heart attack 10 minutes after the test. That is because while the patient may have a history of cardiac symptoms, those symptoms might not have been present when the test was performed. As a result, the test may appear normal in a patient with severe heart disease as it cannot identify a narrowing within an artery unless it is evaluated during physical stress or during a heart attack. Similarly, though the ECG is the definitive test in patients with a rhythm disturbance, if the patient is not in the midst of an arrhythmia when the test is performed, it just may appear normal despite the existence of a serious problem. Hence, by extracting some features from the ECG signal such as amplitude and duration of $\mathrm{P}, \mathrm{QRS}$ and T wave, it can be used as an input vectors for the Hybrid Multilayer Perceptron (HMLP) network to determine the heart abnormality activity accurately. 


\section{LITERATURE REVIEW}

Artificial Neural Network (ANN) is one of the studies of Artificial Intelligence and is a new computing technology in the field of computer science study. ANN also considers the integration of neural networks with another computing method such as fuzzy logic to maximize the interpretation ability of data. Neural networks mostly used for problem solving in pattern recognition, data analysis, control and clustering. ANN has various features including high processing speeds and the ability to learn the solution to a problem from a set of data or examples. Multilayer Perceptron (MLP) [17] is the most suitable and referred neural networks in the pattern recognition detection. This network can be trained to form various decision surfaces in the input space [3].

\subsection{Hybrid Multilayer Perceptron (HMLP)}

An MLP network is a feed-forward artificial neural network that maps a set of input onto a set of appropriate output [4]. The network comprises of an input layer, one or more hidden layer, and an output layer. The number of nodes in the input and output layers is dependent on the number of input and output variables respectively [5]. It has been proven that one hidden layer is sufficient for approximation of continuous function up to a certain acceptable accuracy [6]. The input vector $x_{i}$ is converted to a vector of hidden variables $u_{j}$, using activation function $\varphi_{1}$. The output $u_{j}$ of the $j^{\text {th }}$ node in the hidden layer can be expressed in Equation (1)

$u_{j}=\varphi_{1}\left(\sum_{i=1}^{n} w_{i j} x_{i}+\theta_{j}\right)$

The weight and bias of the connection between the $j^{\text {th }}$ node in the hidden layer and the ith input node are represented by $w_{i j}$ and $\theta_{j}$ respectively. The HMLP network, which is the modified version of MLP, was introduced as an optimized solution that is capable of modeling both linear and non-linear systems [7]. Fig. 1 shows the standard connection for an HMLP network. 


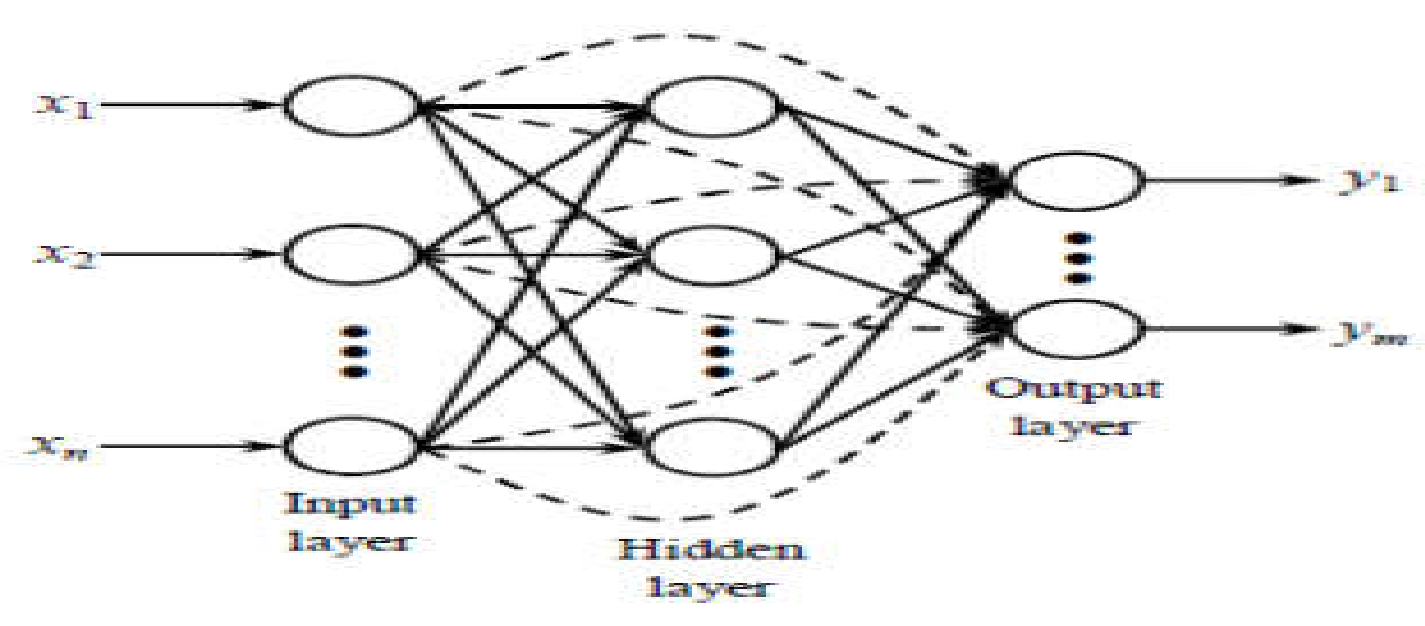

Fig.1. Structure of HMLP network

The network allows the inputs to be connected directly to the output nodes via weighted connections to form a linear model, which is in parallel with the conventional non-linear MLP model [8]. Therefore, the $k^{\text {th }}$ thoutput, $y_{k}$ from the HMLP network can be expressed by Equation (2)

$y_{k}=\varphi_{2}\left(\sum_{j=1}^{h} w_{j k} u_{j}+\sum_{i=1}^{n} w_{i k} x_{i}+\theta_{k}\right)$

\subsection{Electrocardiogram (ECG)}

The electrocardiogram (ECG or EKG) is a medical tool that is usually used to assess and recorded the electrical activity and muscular functions of the heart. Although it is a simple test to perform, a lot amount of training is required for the interpretation of the ECG tracing. The heart has two stage electrical pumps and the heart's electrical activity can be measured by placing electrodes on the skin [9]. The electrocardiogram can measure the rate and rhythm of the heartbeat, and also provide indirect evidence of blood flow to the heart muscle.

There are many type of heartbeat, also refers as rhythm. Normally, the heart beats in a normal (sinus) rhythm as shown in Fig. 2(a) with each electrical impulse generated by the sino-atrail (SA) node resulting in a ventricular contraction, or heartbeat [9]. There are a variety of abnormal electrical rhythms as shown in Fig. 2(b) and 2(c). Some of them are normal and some of them are potentially dangerous. There is also electrical rhythm that does not generate a heartbeat and is the cause of sudden death. 


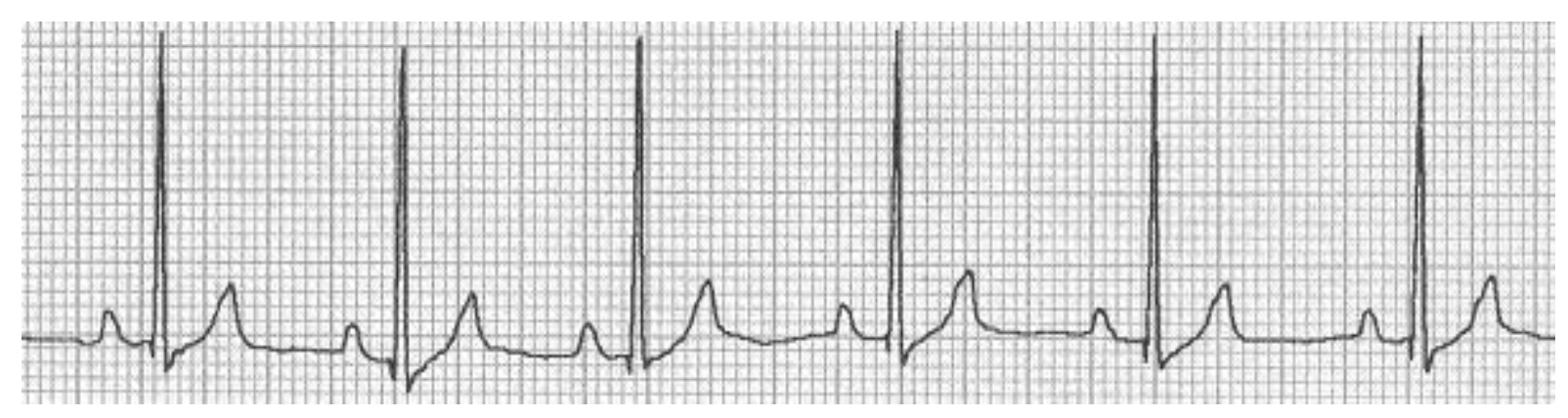

Fig.2(a). Normal ECG signals

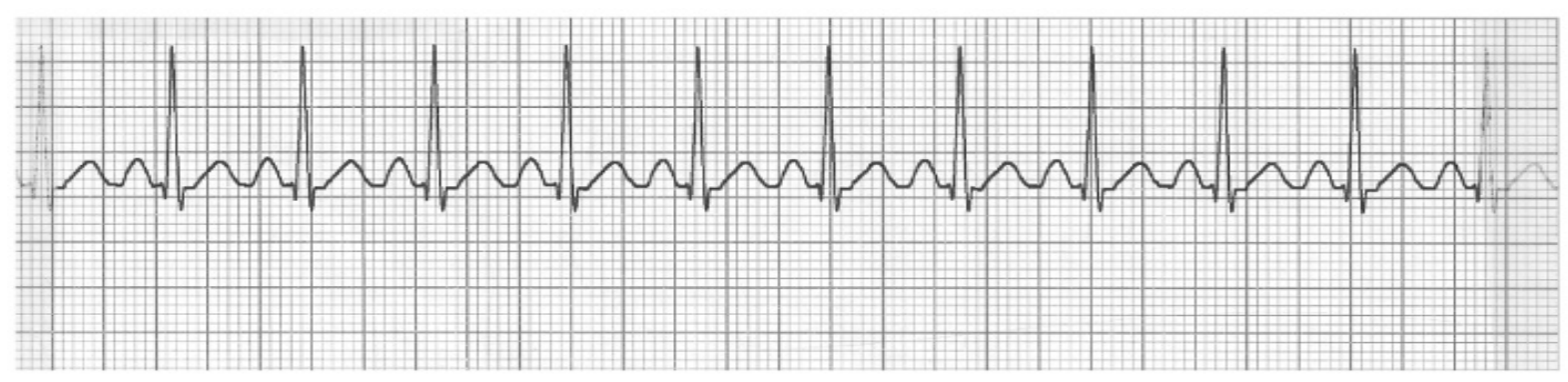

Fig.2(b). Tachycardia ECG signals

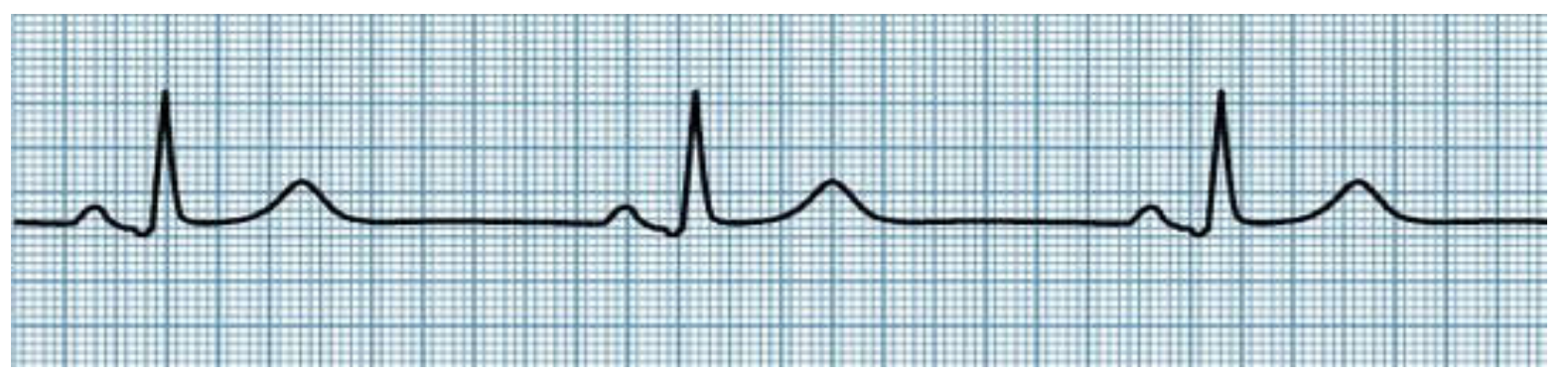

Fig.2(c). Bradycardia ECG signals

To briefly summarize the components of normal ECG readings, it consists of waveform components which indicate electrical events during one heartbeat. These waveforms are labeled P, Q, R, S, T and U [10]. P wave is the first short upward movement of the ECG tracing. It indicates that the atria are contracting, pumping blood into the ventricles. The QRS complex normally beginning with a downward deflection, Q; a larger upwards deflection, a peak (R) and then a downwards $\mathrm{S}$ wave. The QRS complex represents ventricular depolarization and contraction. The PR interval indicates the transit time for the electrical signal to travel from the sinus node to the ventricles. $\mathrm{T}$ wave is normally a modest upwards waveform, representing ventricular repolarization.

\section{METHODOLOGY}

The ECG data for the study was acquired from the Physionet database [11]. The data from 
other classifiers are obtained from [12]. The samples include unprocessed signal for cardiomyopathy disease and other form of arrhythmias. Healthy data were also acquired for control purposes. The ECG signals were all sampled at a frequency of $1 \mathrm{kHz}$. For the purpose of this paper, all signal processing tasks and classification using artificial neural network were performed using MATLAB R2013b. The ECG is exposed to interference from the power lines, electromyogram (EMG) [18] signals and motion artifacts. Hence, it is a requirement for the raw signal to go through a preprocessing stage for noise removal and baseline correction [13].

Recursive prediction error algorithm (RPE) was originally derived by [16] and modified by [15] to train MLP networks [14]. RPE algorithm is a Gauss-Newton type algorithm that will generally provide a better performance than a steepest descent type algorithm such as back propagation algorithm. In nowadays study, the convergence rate of the RPE algorithm is further improved by using the optimized momentum and learning rate. The momentum and learning rate in this research are varied compared to the constant values in [15].

Six features from the ECG data reading will be used as input vectors for the HMLP network. The features are amplitude of $\mathrm{P}$ wave, QRS segment and $\mathrm{T}$ wave. Other features are the duration of $\mathrm{P}$ wave, QRS segment and T wave. This means that the HMLP network will have 6 input nodes to be applied to the network structure. The selection of all this 6 features is based on the changes of the ECG signal from normal reading to abnormal reading. The total data used for each condition is 200 . All the data are inserted to the neural network randomly. The total data used is suitable for training the network. Based on the six features from the ECG signal that are set as an input vector, the neural network is used to classify the heart condition whether it is normal or abnormal. The classification of the heart condition into normal and abnormal are represented as two output nodes as shown on Fig. 3. Each output node functions as a detector for the heart condition. 


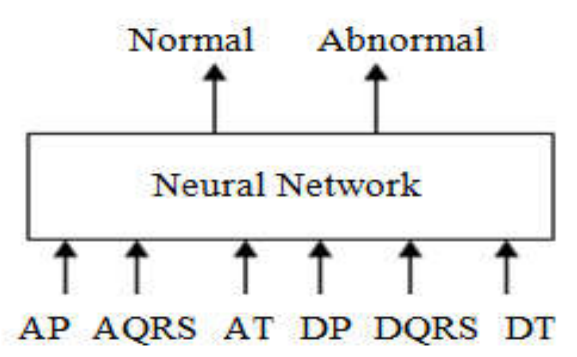

Fig.3. Heart abnormal detection block by using HMLP network

Based on Fig. 3, the input vectors are represented where AP $=$ Amplitude of $\mathrm{P}$ wave, $\mathrm{AQRS}=$ Amplitude of QRS complex, AT = Amplitude of $\mathrm{T}$ wave, $\mathrm{DP}=$ Duration of $\mathrm{P}$ wave, $\mathrm{DQRS}=$ Duration of QRS complex and DT = Duration of T wave.

\section{RESULTS AND DISCUSSION}

The HMLP is examined for its performance, through computational experiment on 6 input parameters that had been extracting from the ECG signal from [11]. The 6 input parameters are amplitude of $\mathrm{P}$ wave, QRS segment, T wave and duration of P wave, QRS segment and T wave. The total data selected is $200 ; 100$ for training purpose and 100 for testing sample. Moreover, the results are compared with MLP network trained by three different training algorithms which are backpropagation (BP), Lavenberg Marquardt (LM) and Bayesian Regularization (BR).

The research applies two different analyses which are the optimum structure analysis becomes the first analysis, which is implemented to determine the optimum number of hidden nodes that can produce the best cardiac abnormality classification. In the analysis, the MLP network is trained with 100 epochs. Table 1 shows the result of the analysis for different types of training algorithm.

Table 1. Performance of optimum structure analysis of HMLP and MLP network trained using different training algorithms

\begin{tabular}{cc}
\hline Neural Network & Optimum Number of Hidden Nodes \\
\hline HMLP & 3 \\
MLP trained by BP & 15 \\
MLP trained by LM & 8 \\
MLP trained by BR & 5 \\
\hline
\end{tabular}


The second analysis, (i.e. the performance comparison analysis) is then applied using the optimum structure of the MLP network obtained in the first analysis. The analysis is done based on the accuracy of correct classifications of heart abnormality. Table 2 shows the result of the analysis for training and testing phase.

Table 2. Performance of accuracy analysis of HMLP and MLP network trained using different training algorithms

\begin{tabular}{cccc}
\hline Neural Network & \multicolumn{3}{c}{ Accuracy Percentage, (\%) } \\
& Training & Testing & Overall \\
\hline HMLP & 96.73 & 95.92 & 96.32 \\
MLP trained by BP & 89.50 & 87.75 & 88.63 \\
MLP trained by LM & 93.25 & 92.50 & 92.88 \\
MLP trained by BR & 93.50 & 92.88 & 93.19 \\
\hline
\end{tabular}

The results show in Table 1 show the MLP network trained by the HMLP network forms the simplest network architecture, as it only requires 3 hidden nodes as compared to other training algorithms (MLP trained by BP, LM and BR). For the performance analysis, the results obtained as shows in Table 2 show that the HMLP network capable to produce the best performance of accuracy with $96.73 \%$ and $95.92 \%$ for training and testing phase respectively which gives overall accuracy of $96.32 \%$.

\section{CONCLUSION}

This paper investigates the capability of neural network to classify the heart abnormality data and the result is compared with the clinical monitoring approach. This paper study proves that the ANN is capable and reliable to classify the heart abnormality based on both amplitude and duration of the $\mathrm{P}, \mathrm{QRS}$ and T peak of ECG signal. This paper also addresses the improvement on neural network structure may improvement the performance of the neural during the optimum structure analysis and the accuracy analysis. 


\section{ACKNOWLEDGEMENTS}

This research work is supported by the (RAGS/1/2014/TK03/UPNM/2) research grant.

\section{REFERENCES}

[1] National Institute of Health (NIH). What cause a heart attack? Maryland: National Heart, Lung, and Blood Institute, 2013

[2] Hashim F R, Soraghan J J, Petropoulakis L, Daud N G. EMG cancellation from ECG signals using modified NLMS adaptive filters. In IEEE Conference on Biomedical Engineering and Sciences, 2014, pp. 735-739

[3] Isa N A, Hashim F R, Mei F W, Ramli D A, Omar W M, Zamli K Z. Predicting quality of river's water based on algae composition using artificial neural network. In IEEE International Conference on Industrial Informatics, 2006, pp. 1340-1345

[4] Ramli D A, Saleh J M, Hashim F R, Isa N A. Multilayered Perceptron (MLP) network trained by recursive least squares algorithm. In 1st IEEE International Conference on Computers, Communications, and Signal Processing with Special Track on Biomedical Engineering, 2005, pp. 288-291

[5] Hashim F R, Soraghan J J, Petropoulakis L. Multi-classify hybrid multilayered perceptron (HMLP) network for pattern recognition applications. In IFIP International Conference on Artificial Intelligence Applications and Innovations, 2012, pp. 19-27

[6] Funahashi K I. On the approximate realization of continuous mappings by neural networks. Neural Networks, 1989, 2(3):183-192

[7] Mat-Isa N A, Mashor M Y, Othman N H. An automated cervical pre-cancerous diagnostic system. Artificial Intelligence in Medicine, 2008, 42(1):1-11

[8] Isa N A, Mamat W M. Clustered-hybrid multilayer perceptron network for pattern recognition application. Applied Soft Computing, 2011, 11(1):1457-466

[9] Hashim F R, Petropoulakis L, Soraghan J, Safie S I. Wavelet based motion artifact removal for ECG signals. In IEEE EMBS Conference on Biomedical Engineering and Sciences, 2012, pp. 339-342

[10] Practical Clinical Skills. ECG interpretation. Pennsylvania: Medical Simulation and 
Training LLC, 2017

[11] Physionet. PhysioBank ATM. York: Physionet, 2017

[12] Eklund P, Hoang A. A performance survey of public domain supervised machine learning algorithms. Australian Journal of Intelligent Information Systems, 2006, 9(1):1-47

[13] Sornmo L., Laguna P. Bioelectrical signal processing in cardiac and neurological applications. Massachusetts: Academic Press, 2005

[14] Mashor M Y. Modified recursive prediction error algorithm for training layered neural network. International Journal of the Computer, the Internet and Management, 2003, 11(2):24-36

[15] Chen S, Gibson G J, Cowan C F, Grant P M. Adaptive equalization of finite non-linear channels using multilayer perceptrons. Signal Processing, 1990, 20(2):107-119

[16] Ljung L., Söderström T. Theory and practice of recursive identification. Massachusetts: MIT Press, 1983

[17] Yassin I M, Jailani R, Ali M, Baharom R, Hassan A, Rizman Z I. Comparison between cascade forward and multi-layer perceptron neural networks for NARX functional electrical stimulation (FES)-based muscle model. International Journal on Advanced Science, Engineering and Information Technology, 2017, 7(1):215-221

[18] Nor M N M, Jailani R, Tahir N M, Yassin I M, Zairi I R, Rahmat H. EMG signals analysis of $\mathrm{BF}$ and RF muscles in autism spectrum disorder (ASD) during walking. International Journal on Advanced Science, Engineering and Information Technology, 2016, 6(5):793-798

\section{How to cite this article:}

Hashim FR, Adnan J, Ibrahim MM, Ishak MT, Din MFM, Daud NGN, Rizman ZI.Heart abnormality detection by using artificial neural network. J. Fundam. Appl. Sci., 2017, 9(3S), $1-10$ 